\title{
Low muscle mass and Charlson comorbidity index are risk factors for short-term postoperative prognosis of elderly patients with gastrointestinal tumor: a cross-sectional study
}

Jiaqiu Wang ${ }^{1,2}$, Liqian Xu ${ }^{1,2}$, Shunmei Huang ${ }^{1,2}$, Quan Hui ${ }^{1,2}$, Xuexue Shi ${ }^{1,2}$ and Qin Zhang ${ }^{1,2^{*}}$

\begin{abstract}
Background: Sarcopenia is one of the most frequent syndromes in older adults and one of its main characteristics is low muscle mass. Gastrointestinal tumor is a malignant disease with high incidence. This study aimed to investigate the risk factors of low muscle mass in older adults with gastrointestinal tumor, the prognostic indicators of and shortterm outcomes after resection for gastrointestinal tumor, and to explore the relationship between low muscle mass and short-term postoperative prognosis.
\end{abstract}

Method: A total of 247 older patients with gastrointestinal tumors who underwent radical resection in 2019 were included in this study. Relevant indexes were calculated using L3 slice image of computed tomography (CT) to evaluate low muscle mass. Short-term postoperative complications and length of stay were considered as short-term outcomes of this study.

Results: Advanced age, lower higher body mass index (BMI), lower hemoglobin, having history of abdominal surgery and higher visceral fat index (VFI) were risk factors of low muscle mass, while higher BMI and lower subcutaneous fat index (SFI) were protective factors of low muscle mass. Further multivariate logistic regression analysis showed that having history of abdominal surgery, advanced age and lower BMI were independent risk factors. Low muscle mass and higher Charlson comorbidity index were independent risk factors of short-term postoperative complications in older adults with gastrointestinal tumor. Higher Charlson comorbidity index gave rise to longer length of stay.

Conclusions: Low muscle mass and higher Charlson comorbidity index predict poor short-term prognosis of older patients undergoing gastrointestinal tumor resection.

Keywords: Low muscle mass, Gastrointestinal tumor, Geriatrics, Complications, Length of stay

*Correspondence: zhangqin1978@zju.edu.cn

${ }^{2}$ Zhejiang Provincial Key Laboratory for Diagnosis and Treatment of Aging and Physic-chemical Injury Diseases, The First Affiliated Hospital, Zhejiang University School of Medicine, 79 Qingchun Raod, Hangzhou, Zhejiang 310003, People's Republic of China

Full list of author information is available at the end of the article

\section{Introduction}

With the aging tendency of global population, the number of people with low muscle mass is increasing. Low muscle mass is one of the main characteristics of sarcopenia which is one of the most frequent syndromes in older adults. As newly released guideline, sarcopenia is defined as the decline in muscle quality, strength and 
function which are relevant with aging [1]. Older adults with sarcopenia have a higher risk of falls, fractures, and motor functional decline. In recent years, more and more studies on low muscle mass have been conducted to help explore sarcopenia, and guidelines about sarcopenia have been constantly updated at home and abroad to help clinical work and related scientific research. As previous research showed, nearly $1 / 10$ older adults suffered with muscle quality decline [2], so that the society should attach more importance to sarcopenia.

Solid tumor of gastrointestinal tract is one of the malignant diseases of high incidence. According to the GLOBOCAN 2018 data released by the World Health Organization (WHO) in 2018, colorectal cancer ranks the third and gastric cancer the fourth among the top ten tumors in terms of incidence [3]. There are about 1.4 million new cases of colorectal cancer and 1 million new cases of stomach cancer per year globally, and about $70 \%$ of stomach cancer occurs in developing countries [4]. In China, there are a large number of patients with colorectal tumor and stomach tumor, and the effective treatment for most of them potentially is surgical resection. Older adults with cancer have a higher incidence of sarcopenia [4]. When both cancer and sarcopenia happen to older patients, it may increase the clinical adverse events, accelerate malignancy process and further influence the survival of older patients $[4,5]$. Recent studies have showed that low muscle mass is correlated with poor prognosis after resection surgery for colorectal cancer and stomach cancer [6, 7]. However, relevant research in the Chinese population is still limited.

Therefore, this study explored the relationship between low muscle mass, common clinical indicators and shortterm postoperative complications in older patients with colorectal cancer and gastric cancer. We aimed to further identify prognostic indicators for older patients with resection and to intervene precisely.

\section{Method \\ Patients}

Older patients undergoing resection for gastric cancer or colorectal cancer at the First Affiliated Hospital of Zhejiang University from January to December in 2019 were included in this cross-sectional study. Inclusion criteria were: 1 . patients' age $\geq 70$ years old; 2 . Abdominal CT were completed within 15 days before the surgery; 3. Postoperative pathology confirmed malignant tumor. Patients with incomplete important data, such as leukocyte, hemoglobin, neutrophils to lymphocytes ratio (NLR), albumin, height, weight, postoperative course record and etc., were excluded from this study.

All the data were collected by experienced geriatrician from electronic medical records, which included: basic information such as sex, height, weight, age and etc.; comorbidities were evaluated by Charlson comorbidity index, the gastrointestinal tumor treated in this hospital stay was not evaluated as comorbidity [8]; history of abdominal surgery related to malignancy or benign diseases; history of alcohol, tobacco; laboratory parameters included leukocyte, hemoglobin, albumin and NLR within 15 days before the surgery; CT slice at L3; the operation data included the duration of operation, intraoperative blood loss, and postoperative pathology; cancer stage which was based on The Union for International Cancer Control (UICC) cancer tumor node metastasis (TNM) staging system (8th edition) [9]; and postoperative outcomes included postoperative complications which was evaluated by Clavien-Dindo classification [10] and length of stay.

\section{Imaging analysis}

The CT slice at L3 was analysed by Image J (NIH Image J version 1.52a). Referred to the former research, skeletal muscle threshold is $-29 \mathrm{HU}$ to $150 \mathrm{HU}$ and the adipose tissue threshold is $-190 \mathrm{HU}$ to $-30 \mathrm{HU}$ [6]. An example is shown in Fig. 1, in which the red part represents muscle tissue, the dark green part represents subcutaneous fat tissue, and the light green part represents visceral fat tissue. This study measured the skeletal muscle area (SMA), subcutaneous fat areas (SFA) and visceral fat area (VFA). Obtained area values were divided by the square of the patient's height $\left(\mathrm{m}^{2}\right)$ to get skeletal muscle index (SMI), SFI and VFI. Visceral-to-subcutaneous ratio of fat area (VSR) was also calculated as a parameter. According to the previous large sample Chinese population study, male $\mathrm{SMI} \leq 40.8 \mathrm{~cm}^{2} / \mathrm{m}^{2}$ and female $\mathrm{SMI} \leq 34.9 \mathrm{~cm}^{2} / \mathrm{m}^{2}$ were defined as low muscle mass [11].

\section{Statistics}

Data are given as means (with standard deviation) or medians (with interquartile range). Univariate and multivariate logistic regression were performed to analyze the related variables of postoperative complications. Results were showed as odds ratios (ORs) with 95\% confidence intervals (CIs). Linear regression was performed to analyze the related factors for length of stay. IBM SPSS 25 was used for all this analysis.

\section{Results}

\section{Patients characteristics}

The characteristics of included patients were presented in Table 1. A total of 247 older patients undergoing gastrointestinal tumor surgery were included in this study, 163 of them were men. Ninety-eight patients were suffering from gastric cancer and the rest from colorectal cancer. Their average age was about 75.5 and their average BMI 


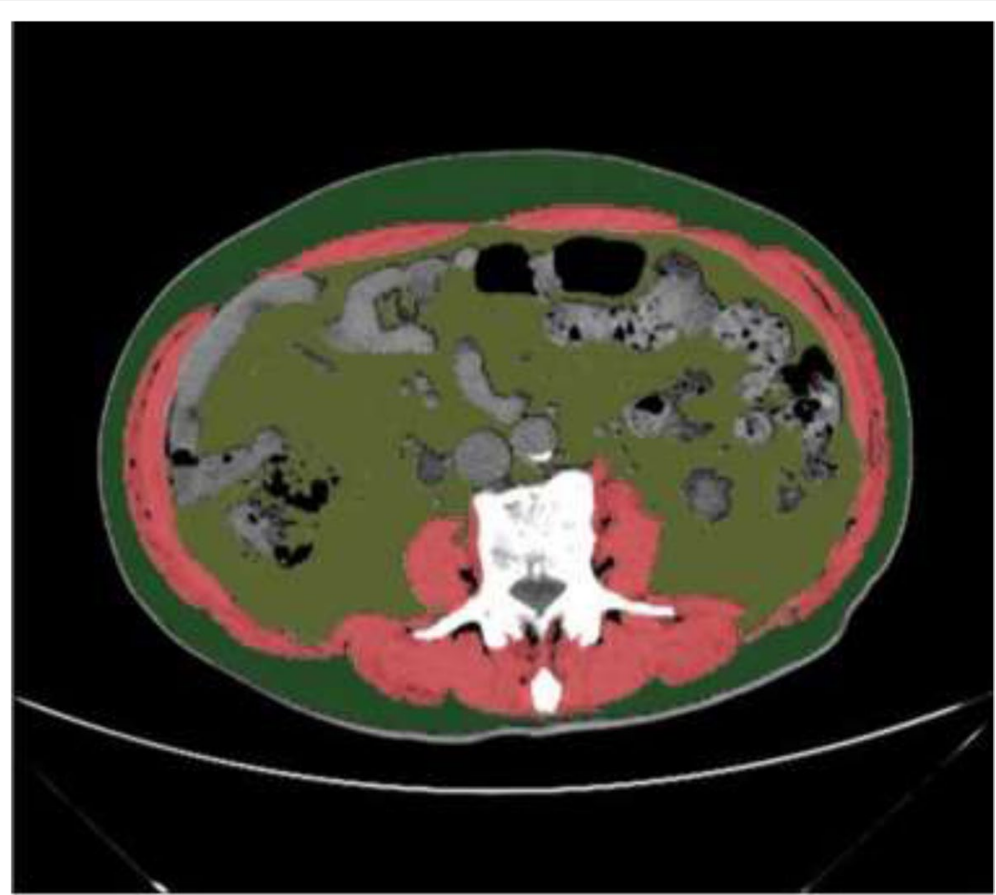

Fig. 1 Skeletal muscle and fat tissue in L3

was around $22.2 \mathrm{~kg} / \mathrm{m}^{2}$. Fifty-two patients had history of alcohol and 68 patients had history of tobacco and most of them were male. Sixty patients had history of abdominal surgery. The mean values of leukocyte, hemoglobin, albumin and NLR were within the range of normal clinical values. Most of the patients were in TNM stage I and II, but only 13 patients were defined as well differentiated histologic type.

Using the Image software, we calculated the parameters of human body composition. SMI, SFI, VFI and VSR were $42.6 \pm 10.9 \mathrm{~cm}^{2} / \mathrm{m}^{2}, \quad 37.6 \pm 24.3 \mathrm{~cm}^{2} / \mathrm{m}^{2}$, $49.4 \pm 35.7 \mathrm{~cm}^{2} / \mathrm{m}^{2}, 1.5 \pm 0.8$.

\section{Factors associated with low muscle mass}

Seventy-one patients were classified as low muscle mass. The incidence rate of low muscle mass in this study was about $28.7 \%$. Twenty-two of them (31.0\%) were with gastric tumor and 49 (69.0\%) were with colorectal tumor.

This study detected several factors associated with low muscle mass as Table 2 showed. In univariate analysis, low muscle mass was associated with advanced age $(p<0.001)$, lower BMI $(p<0.001)$, lower hemoglobin $(p=0.009)$, having history of abdominal surgery $(p=0.010)$, and higher VFI $(p<0.001)$. Higher BMI $(p<0.001)$ and lower SFI $(p=0.005)$ were protective factors for low muscle mass. Multivariate logistic regression was used to further analyze the data and it showed that having history of abdominal surgery (OR:2.5; 95\% CI:1.3 to $5.3 ; p=0.007)$, advanced age (OR:3.0; $95 \% \mathrm{CI}: 1.6$ to 5.7; $p=0.001$ ) and lower BMI (OR:3.4; 95\% CI:1.3 to 8.6; $p=0.011$ ) were independent risk factors for low muscle mass.

\section{Relationship between low muscle mass and postoperative complication}

About 62 patients had grade two and above of ClavienDindo classification of surgical complications. The main complications included postoperative hemorrhage, pulmonary infection, abdominal infection, seroperitoneum, delirium, anastomotic leakage, ileus, venous thrombosis, heart failure, etc. Exploratory laparotomy was performed in four patients after operation because of the postoperative hemorrhage or ileus. One patient got interventional operation for multiple thrombosis. One patient died because of hemorrhoea. One patient discharged from hospital giving up treatment because of severe infection and bone marrow suppression.

Several factors were found associated with postoperative complications as Table 3 presented. In univariate analysis, advanced age $(p=0.012)$, higher Charlson comorbidity index $(p=0.014)$, and low muscle mass $(p=0.001)$ were associated with postoperative complications. Further multivariate logistic regression analysis showed that low muscle mass (OR:2.6; 95\% CI:1.4 to $4.9 ; p=0.002$ ), and higher Charlson comorbidity 
Table 1 Overall characteristics of patients

\begin{tabular}{|c|c|c|c|}
\hline & gastric cancer $(n=99)$ & colorectal cancer $(n=148)$ & Total $(n=247)$ \\
\hline Age (years) & $74.6(4.1)$ & $76.1(5.1)$ & $75.5(4.8)$ \\
\hline Male, n(\%) & $77(77.8)$ & $86(58.1)$ & 163(65.6) \\
\hline BMI $\left(\mathrm{kg} / \mathrm{m}^{2}\right)$ & $21.8(2.9)$ & $22.5(3.4)$ & $22.2(3.2)$ \\
\hline Charlson comorbidity index & $4(1)$ & $4(2)$ & $4(2)$ \\
\hline History of alcohol, n(\%) & $28(28.2)$ & $24(16.2)$ & $52(21.1)$ \\
\hline History of tobacco, n(\%) & $40(40.4)$ & 28(18.9) & $68(27.5)$ \\
\hline History of abdominal surgery, n(\%) & 15(15.1) & $45(30.4)$ & $60(24.3)$ \\
\hline \multicolumn{4}{|l|}{ Major laboratory indicators } \\
\hline Leukocyte $(\times 10 \wedge 9 / L)$ & $5.4(2.0)$ & $5.9(2.2)$ & $5.7(2.1)$ \\
\hline Hemoglobin(g/L) & $125.0(28.8)$ & 129.0(33.1) & $126.5(32.0)$ \\
\hline Albumin(g/L) & $39.7(8.2)$ & $43.0(5.4)$ & $42.1(6.7)$ \\
\hline NLR & $2.3(1.8)$ & 2.4(1.4) & $2.4(1.5)$ \\
\hline Intraoperative blood loss (mL) & 132.8(81.9) & 107.6(93.1) & 116.3(89.0) \\
\hline Duration of operation (min) & $202.0(67.0)$ & 180.0(63.0) & $188(65)$ \\
\hline \multicolumn{4}{|l|}{ Cancer stage, n(\%) } \\
\hline । & $56(56.6)$ & $42(28.4)$ & 98(39.7) \\
\hline$\|$ & $32(32.3)$ & $51(34.5)$ & 83(33.6) \\
\hline III & 11(11.1) & $55(37.2)$ & $66(26.7)$ \\
\hline \multicolumn{4}{|l|}{ Histologic type, n(\%) } \\
\hline Poorly differentiated & $63(63.6)$ & $36(24.3)$ & $99(40.1)$ \\
\hline Moderately differentiated & 29(29.3) & 106(71.6) & $135(54.7)$ \\
\hline Well differentiated & $7(7.1)$ & $6(4.1)$ & $13(5.3)$ \\
\hline \multicolumn{4}{|l|}{ Body composition } \\
\hline $\mathrm{SMI}\left(\mathrm{cm}^{2} / \mathrm{m}^{2}\right)$ & $44.7(9.4)$ & $40.9(10.7)$ & $42.6(10.9)$ \\
\hline $\mathrm{SFI}\left(\mathrm{cm}^{2} / \mathrm{m}^{2}\right)$ & $34.5(19.2)$ & $39.7(27.6)$ & $37.6(24.3)$ \\
\hline VFI $\left(\mathrm{cm}^{2} / \mathrm{m}^{2}\right)$ & $45.7(28.6)$ & $50.7(41.0)$ & $49.4(35.7)$ \\
\hline VSR & $1.3(0.7)$ & $1.2(0.8)$ & $1.5(0.8)$ \\
\hline Low muscle mass, n(\%) & $22(22.2)$ & 49(33.1) & $71(28.7)$ \\
\hline Postoperative complication, n(\%) & $14(14.1)$ & $43(29.1)$ & $62(25.1)$ \\
\hline Length of stay (day) & 10(3) & $8(4)$ & $9(5)$ \\
\hline
\end{tabular}

$B M I$ body mass index, NLR neutrophil to lymphocyte ratio, SMI skeletal muscle index, SFI subcutaneous fat index, VFI visceral fat index, VSR visceral to subcutaneous ratio of fat area

index (OR:2.1; 95\% CI:1.1 to $3.9 ; p=0.026$ ) were independent risk factors of postoperative complications.

\section{Factors associated with length of stay}

Linear regression was employed to analyze the risk factors of length of stay. After the factors which were correlated with each other were excluded, it was found that Charlson comorbidity index $(p=0.019)$, tumor site $(p=0.016)$, and duration of surgery $(p=0.045)$ were significantly correlated with length of hospital stay. Higher Charlson comorbidity index and longer operative time will result in longer hospital stays. The length of stay of patient with gastric cancer was significantly longer than those who with colorectal cancer.

\section{Discussion}

This study mainly investigated the risk factors of low muscle mass in older patients with digestive tract tumors and the relationship between low muscle mass and shortterm postoperative outcome. This study found that the incidence of low muscle mass in older patients with gastric cancer or colorectal cancer was about $28.7 \%$, which was clearly associated with history of abdominal surgery, lower BMI, and advanced age. The occurrence of postoperative complications was correlated with low muscle mass and higher Charlson comorbidity index.

The Asian Working Group for Sarcopenia (AWGS) released the latest expert consensus on the diagnosis and treatment of sarcopenia in 2019. As the expert consensus suggested, the commonly used methods for muscle 
Table 2 Univariate and multivariate logistic regression analysis of low muscle mass associated factors

\begin{tabular}{|c|c|c|c|c|c|c|}
\hline \multirow[t]{2}{*}{ Variable } & \multicolumn{3}{|c|}{ Univariate analysis } & \multicolumn{3}{|c|}{ Multivariate analysis } \\
\hline & OR & $95 \% \mathrm{Cl}$ & $p$ Value & OR & $95 \% \mathrm{Cl}$ & $p$ Value \\
\hline Sex $x^{a}$ & 0.6 & $0.3-1.1$ & 0.084 & & & \\
\hline Age $^{\mathrm{b}}$ & 3.2 & $1.8-5.8$ & $<0.001$ & 3.0 & $1.6-5.7$ & 0.001 \\
\hline Cancer site ${ }^{c}$ & 0.6 & $0.3-1.1$ & 0.078 & & & \\
\hline$B M l^{d}$ & 3.3 & $1.3-8.0$ & 0.009 & 3.4 & $1.3-8.6$ & 0.011 \\
\hline$B M d^{d^{*}}$ & 0.2 & $0.1-0.4$ & $<0.001$ & 0.2 & $0.1-0.4$ & $<0.001$ \\
\hline Charlson comorbidity index ${ }^{\mathrm{e}}$ & 1.5 & $0.8-2.7$ & 0.207 & & & \\
\hline History of alcohol ${ }^{f}$ & 1.7 & $0.8-3.1$ & 0.086 & & & \\
\hline History of tobacco ${ }^{9}$ & 1.5 & $0.8-2.8$ & 0.252 & & & \\
\hline History of abdominal surgery ${ }^{h}$ & 2.2 & $1.2-4.1$ & 0.010 & 2.6 & $1.3-5.3$ & 0.007 \\
\hline Leukocyte & 1.4 & $0.8-2.4$ & 0.254 & & & \\
\hline Hemoglobin ${ }^{j}$ & 2.1 & $1.2-3.7$ & 0.009 & & & \\
\hline Albumin ${ }^{k}$ & 1.7 & $0.9-2.9$ & 0.075 & & & \\
\hline$N L R^{\prime}$ & 0.7 & $0.4-1.2$ & 0.159 & & & \\
\hline Cancer stage $^{m}$ & 1.1 & $0.8-1.5$ & 0.470 & & & \\
\hline Histologic type ${ }^{n}$ & 1 & $0.6-1.7$ & 0.860 & & & \\
\hline$\left.\mathrm{SFl}\right|^{\circ}$ & 0.4 & $0.3-0.8$ & 0.005 & & & \\
\hline$V F I^{p}$ & 2.9 & $1.6-5.3$ & $<0.001$ & & & \\
\hline VSR $R^{q}$ & 1.0 & $0.6-1.7$ & 0.985 & & & \\
\hline
\end{tabular}

OR odds ratio, $\mathrm{Cl}$ confidence interval, $B M I$ body mass index, NLR neutrophil to lymphocyte ratio, $S M I$ skeletal muscle index, SFI subcutaneous fat index, $\mathrm{VFI}$ visceral fat index, VSR visceral to subcutaneous ratio of fat area

a female as reference

${ }^{b}$ age $\leq 75$ as reference

${ }^{c}$ colorectal cancer as reference

${ }^{d}$ BMl $<18.5 \mathrm{~kg} / \mathrm{m}^{2}$ vs $18.5 \mathrm{~kg} / \mathrm{m}^{2} \leq \mathrm{BMI} \leq 24 \mathrm{~kg} / \mathrm{m}^{2}$

$\mathrm{d}^{*} \mathrm{BMl}>24 \mathrm{~kg} / \mathrm{m}^{2}$ vs $18.5 \mathrm{~kg} / \mathrm{m}^{2} \leq \mathrm{BMI} \leq 24 \mathrm{~kg} / \mathrm{m}^{2}$

${ }^{e}$ Charlson comorbidity index $\leq 4$ as reference

${ }^{f}$ no history of alcohol as reference

${ }^{g}$ no history of tobacco as reference

${ }^{\mathrm{h}}$ no history of abdominal surgery as reference

' leukocyte $\geq 5.7 \times 10^{\wedge} 9 / \mathrm{L}$ as reference

${ }^{j}$ hemoglobin $\geq 126.5 \mathrm{~g} / \mathrm{L}$ as reference

$\mathrm{k}$ albumin $\geq 42.1 \mathrm{~g} / \mathrm{L}$ as reference

${ }^{\prime} \mathrm{NLR} \geq 2.4$ as reference

$\mathrm{m}$ stagell and stagelll vs stagel

${ }^{\mathrm{n}}$ moderately and well differentiated vs poorly differentiated

${ }^{\circ} \mathrm{SFI} \leq 37.6 \mathrm{~cm}^{2} / \mathrm{m}^{2}$ as reference

$\mathrm{p} \mathrm{VFI} \leq 49.4 \mathrm{~cm}^{2} / \mathrm{m}^{2}$ as reference

${ }^{\mathrm{q}} \mathrm{VSR} \leq 1.45$ as reference

mass measurement are DXA or BIA, and CT is also recognized as a good method for skeletal muscle mass measurement especially for assessing muscle volume [1, 12]. However, there is some debates on the diagnostic threshold. In general studies, L3 plane was selected to calculate the muscle area and SMI value. Low muscle mass was defined by the cut-off value of SMI. A study published in The Lancet Oncology in 2008 suggested that men with $\mathrm{SMI}<52.4 \mathrm{~cm}^{2} / \mathrm{m}^{2}$ and women with $\mathrm{SMI}<38.5 \mathrm{~cm}^{2} / \mathrm{m}^{2}$ were considered to have CT-assessed sarcopenia [13].
This cut-off value is frequently used. Different ethnic groups suit different SMI cut-off value and some studies chose more complicated cut-off values according to both BMI value and SMI value [14]. Since the object of this study was Asian population, and there is a big difference in physique between the western population and Asian population, we referred to a dependable study of a large sample of Chinese population in which men with $\mathrm{SMI} \leq 40.8 \mathrm{~cm}^{2} / \mathrm{m}^{2}$ and women with $\mathrm{SMI} \leq 34.9 \mathrm{~cm}^{2} /$ $\mathrm{m}^{2}$ were considered to have low muscle mass [11]. The 
Table 3 Univariate and multivariate logistic regression analysis of postoperative complications associated factors

\begin{tabular}{|c|c|c|c|c|c|c|}
\hline \multirow[t]{2}{*}{ Variable } & \multicolumn{3}{|c|}{ Univariate analysis } & \multicolumn{3}{|c|}{ Multivariate analysis } \\
\hline & OR & $95 \% \mathrm{Cl}$ & $p$ Value & OR & $95 \% \mathrm{Cl}$ & $p$ Value \\
\hline $\operatorname{Sex}^{\mathrm{a}}$ & 1.4 & $0.8-2.6$ & 0.226 & & & \\
\hline $\mathrm{Age}^{\mathrm{b}}$ & 2.1 & $1.2-3.8$ & 0.012 & & & \\
\hline Cancer site $^{c}$ & 1.7 & $0.9-3.1$ & 0.095 & & & \\
\hline $\mathrm{BMI}^{\mathrm{d}}$ & 2.2 & $0.9-5.4$ & 0.097 & & & \\
\hline $\mathrm{BMI}^{\mathrm{d}^{*}}$ & 0.8 & $0.4-1.6$ & 0.618 & & & \\
\hline Charlson comorbidity index & 2.2 & $1.2-4.0$ & 0.014 & 2.1 & $1.1-3.9$ & 0.026 \\
\hline History of alcoholf & 2.2 & $0.9-4.9$ & 0.067 & & & \\
\hline History of tobacco ${ }^{9}$ & 1.1 & $0.6-2.1$ & 0.723 & & & \\
\hline History of abdominal surgery ${ }^{h}$ & 1.8 & $1.0-3.5$ & 0.065 & & & \\
\hline Leukocyte $^{i}$ & 1.3 & $0.7-2.3$ & 0.400 & & & \\
\hline Hemoglobin & 1.2 & $0.7-2.1$ & 0.207 & & & \\
\hline Albumink & 0.9 & $0.5-1.7$ & 0.851 & & & \\
\hline$N L R^{l}$ & 0.7 & $0.4-1.2$ & 0.207 & & & \\
\hline Cancer stage $^{m}$ & 1.8 & $1.0-3.3$ & 0.060 & & & \\
\hline Histologic type $^{n}$ & 1.2 & $0.7-1.9$ & 0.510 & & & \\
\hline Low muscle mass ${ }^{\circ}$ & 2.7 & $1.5-5.0$ & 0.001 & 2.6 & $1.4-4.9$ & 0.002 \\
\hline SFI & 0.8 & $0.5-1.4$ & 0.442 & & & \\
\hline $\mathrm{VFI} \mathrm{I}^{\mathrm{a}}$ & 0.9 & $0.5-1.6$ & 0.696 & & & \\
\hline$V_{S R}^{r}$ & 1.4 & $0.8-2.4$ & 0.288 & & & \\
\hline Intraoperative blood loss ${ }^{s}$ & 1.4 & $0.7-2.6$ & 0.386 & & & \\
\hline Duration of operation ${ }^{\mathrm{t}}$ & 1.8 & $1.0-3.2$ & 0.051 & & & \\
\hline
\end{tabular}

OR odds ratio, CI confidence interval, BMI body mass index, NLR neutrophil to lymphocyte ratio, SMI skeletal muscle index, SFI subcutaneous fat index, VFI visceral fat index, VSR visceral to subcutaneous ratio of fat area

a female as reference

${ }^{b}$ age $\leq 75$ as reference

' colorectal cancer as reference

${ }^{\mathrm{d}} \mathrm{BMl}<18.5 \mathrm{~kg} / \mathrm{m}^{2}$ vs $18.5 \mathrm{~kg} / \mathrm{m}^{2} \leq \mathrm{BMl} \leq 24 \mathrm{~kg} / \mathrm{m}^{2}$

$\mathrm{d}^{*} \mathrm{BMI}>24 \mathrm{~kg} / \mathrm{m}^{2}$ vs $18.5 \mathrm{~kg} / \mathrm{m}^{2} \leq \mathrm{BMI} \leq 24 \mathrm{~kg} / \mathrm{m}^{2}$

${ }^{e}$ Charlson comorbidity index $\leq 4$ as reference

${ }^{f}$ no history of alcohol as reference

${ }^{9}$ no history of tobacco as reference

${ }^{\mathrm{h}}$ no history of abdominal surgery as reference

' leukocyte $\geq 5.7 \times 10^{\wedge} 9 / \mathrm{L}$ as reference

${ }^{j}$ hemoglobin $\geq 126.5 \mathrm{~g} / \mathrm{L}$ as reference

${ }^{k}$ albumin $\geq 42.1 \mathrm{~g} / \mathrm{L}$ as reference

${ }^{\prime} \mathrm{NLR} \geq 2.4$ as reference

$\mathrm{m}$ stagell and stagelll vs stagel

${ }^{n}$ moderately and well differentiated vs poorly differentiated

${ }^{\circ}$ without low muscle mass as reference

$\mathrm{p} \mathrm{SFI} \leq 37.6 \mathrm{~cm}^{2} / \mathrm{m}^{2}$ as reference

${ }^{\mathrm{q}} \mathrm{VFI} \leq 49.4 \mathrm{~cm}^{2} / \mathrm{m}^{2}$ as reference

${ }^{r} \mathrm{VSR} \leq 1.45$ as reference

${ }^{s}$ blood loss $\leq 116 \mathrm{~mL}$ as reference

${ }^{\mathrm{t}}$ time $\leq 196 \mathrm{~min}$ as reference

prevalence of low muscle mass in older adults with cancer was higher than in which without cancer according to previous researches [5]. In this study, 71 patients $(28.7 \%)$ were considered with low muscle mass.
Whether low muscle mass is a risk factor of short-term postoperative complications in older patients is still controversial. Some studies showed that the surgical complications of patients with oesophageal cancer had no 
relation with low muscle mass [15], while in some other cancer such as lung cancer and renal cell carcinoma, low muscle mass seemed to be related with prognosis of surgery $[16,17]$. This study suggested that low muscle mass was an independent risk factor of short-term postoperative complications in older patients with gastric cancer or colorectal cancer, and confirmed that low muscle mass is an important indicator of postoperative prognosis which suggests the necessity of preoperative diagnosis of low muscle mass. This research did not show any relationship between tumor stage, histologic type and surgical prognosis. A larger sample size may be needed for further clarification. In the multivariate logistic regression analysis, this research also showed that higher Charlson comorbidity index was risk factors for short-term surgical complications. A previous study showed that Charlson comorbidity index is an independent risk factor of short- and long-term mortality in hospitalized elderly patients and another one suggested that higher Charlson comorbidity index is related with postoperative complication and longer length of stay in patients with colorectal carcinoma [18]. This study was consistent with the previous research results. Low muscle mass was not found associated with the length of stay, while higher Charlson comorbidity index was a risk factor for longer length of stay, suggesting that patients with more underlying diseases should be taken better postoperative care.

Among the several factors related to low muscle mass found in this study, lower BMI and advanced age have been well discussed and recognized in previous studies [19]. But this research also found that higher BMI was a protective factor for low muscle mass, which may be somewhat controversial. Recently, many studies have come to a conclusion that obesity is a risk factor for low muscle mass as well, and obesity sarcopenia became a hot research topic [1]. However, there also have been many papers suggesting that obesity is not associated with low muscle mass [20], and obesity sarcopenia was not made a clear definition and diagnosis in the newly released sarcopenia guideline [1]. This study found that higher VFI is a risk factor for low muscle mass, while higher SFI is a protective factor for low muscle mass, that is, fat in different parts of the body has different effects on low muscle mass. Some research suggested that abdominal obesity is associated with the development of low muscle mass and parameter VSR is used as a parameter to define abdominal obesity [21]. VSR reflects differences in fat distribution, but cannot reflects the volume of fat. This study did not find any correlation between VSR and low muscle mass or postoperative outcomes. The value of VSR in low muscle mass needs further research efforts. The relationship between obesity and low muscle mass is still controversial and the mechanism is still unknown, which needs to be clarified by further studies. This study also found that the occurrence of low muscle mass was associated with a prior history of abdominal surgery which was not often mentioned in other studies. Abdominal surgery may lead to the functional decline of digestive system and results in emaciation.

This study had some limitations. The length of stay of patients with gastric cancer was found longer than those who with colorectal cancer, which may be because that gastric cancer and colorectal cancer have different surgical process and operative trauma. Cancer site was not found as a risk factor for low muscle mass which might be inconsistent as some clinical studies showed that patients with gastric cancer might have more weight loss than patients with colorectal cancer [22]. Since cancer cachexia is more severe in advanced stage and might not apparent in the early stage [23], and the ratio of patients in advanced stage in this research is not high, the result was not surprising and a larger sample size is needed. This study made analysis in patients with mixed gastrointestinal cancer but not separately, since the two kinds of tumor share some similarities in their characteristics. If with larger sample size, there should be further analysis separately in different cancer site and cancer stage. There was no follow-up of the long-term prognosis of the patients, such as long-term complications and quality of postoperative life. Due to the incompleteness of preoperative surgical examination, some possible relevant nutritional indicators, such as prealbumin, were not included in this study. Our team will continue investigating the subject.

\section{Conclusions}

Age, lower BMI, and history of abdominal surgery are independent risk factors of low muscle mass in the older patients with gastrointestinal tumor. Low muscle mass and Charlson comorbidity index can predict the shortterm prognosis of older patients undergoing gastrointestinal tumor resection. Precise preoperative evaluation is needed for the older patients with gastrointestinal tumor, and more attentive care should be taken to those with low muscle mass and higher Charlson comorbidity index to avoid short-term postoperative complications.

\footnotetext{
Abbreviations

CT: Computed tomography; BMI: Body mass index; VFI: Visceral fat index; SFI: Subcutaneous fat index; WHO: World Health Organization; NLR: Neutrophils to lymphocytes ratio; UICC: Union for International Cancer Control; TNM: Tumor node metastasis; SMA: Skeletal muscle area; SFA: Subcutaneous fat areas; VFA: Visceral fat area; SMI: Skeletal muscle index; VSR: Visceral-to-subcutaneous ratio of fat area; OR: Odds ratio; Cl: Confidence interval; AWGS: Asian Working Group for Sarcopenia.
} 


\section{Acknowledgements}

We sincerely thank Zhang Bao for his help of the method of imaging analysis. Thank Yongfeng Ding and Ye Zhao for their help about the method of statistical analysis.

\section{Authors' contributions}

JW: project development, data analysis, manuscript writing. LX: project development, data analysis. S H: data analysis, manuscript editing. Q H: data collection. X S: data collection. Q Z: project development, manuscript editing. All authors have read and approved this manuscript.

\section{Funding}

This work was funded by National Key Research and Development Program of China (2018YFC200301) and Opening Project of Zhejiang Provincial Top Key Discipline of Pharmaceutical Sciences (CN) (2017-XK-A31). The two program mainly subsidized labor costs and publishing fee.

\section{Availability of data and materials}

The data in this study is not publicly available since national juridical restrictions, but further description or analysis of data are available from authors with reasonable request.

\section{Declarations}

\section{Ethics approval}

The study protocol has got written approval of the Ethics Committee of the first affiliated hospital of Zhejiang university school of medicine. This study was cross-sectional and all the data were anonymous, so the requirement for the informed consent was waived. All methods were performed in accordance with the relevant guidelines and regulations.

\section{Consent for publication}

Not applicable.

\section{Competing interests}

The authors declare that they have no conflicts of interest to this work.

\section{Author details}

'Department of Geriatrics, The First Affiliated Hospital, Zhejiang University School of Medicine, 79 Qingchun Raod, Hangzhou, Zhejiang 310003, People's Republic of China. ${ }^{2}$ Zhejiang Provincial Key Laboratory for Diagnosis and Treatment of Aging and Physic-chemical Injury Diseases, The First Affiliated Hospital, Zhejiang University School of Medicine, 79 Qingchun Raod, Hangzhou, Zhejiang 310003, People's Republic of China.

\section{Received: 5 July 2021 Accepted: 26 November 2021}

\section{Published online: 23 December 2021}

\section{References}

1. Chen L, Woo J, Assantachai P, Auyeung T, et al. Asian working Group for Sarcopenia: 2019 consensus update on sarcopenia diagnosis and treatment. J Am Med Dir Assoc. 2020;21 (3):300-7.e2. https://doi.org/10.1016/j.jamda. 2019.12.012.

2. Makizako H, Nakai Y, Tomioka K, et al. Prevalence of sarcopenia defined using the Asia working Group for Sarcopenia criteria in Japanese community-dwelling older adults: a systematic review and meta-analysis. Phys Ther Res. 2019;22(2):53-7. https://doi.org/10.1298/ptr.R0005.

3. Bray F, Ferlay J, Soerjomataram I, et al. Global cancer statistics 2018: GLOBOCAN estimates of incidence and mortality worldwide for 36 cancers in 185 countries. CA Cancer J Clin. 2018;68(6):394-424. https://doi.org/10.3322/ caac.21492.s.

4. Ryan AM, Power DG, Daly L, et al. Cancer-associated malnutrition, cachexia and sarcopenia: the skeleton in the hospital closet 40 years later. Proc Nutr Soc. 2016;75(2):199-211. https://doi.org/10.1017/S002966511500419X.

5. Rier HN, Jager A, Sleijfer S, et al. The prevalence and prognostic value of low muscle mass in cancer patients: a review of the literature. Oncologist. 2016;21(11):1396-409. https://doi.org/10.1634/theoncologist.2016-0066.
6. Zhang Y, Wang JP, Wang XL, et al. Computed tomography-quantified body composition predicts short-term outcomes after gastrectomy in gastric cancer. Curr Oncol. 2018;25(5):E411-22. https://doi.org/10.3747/co.25.4014.

7. Deng $C$, Lin Y, Wu JS, Cheung Y, et al. Progressive sarcopenia in patients with colorectal Cancer predicts survival. Am J Roentgenol. 2018;210(3):526-32. https://doi.org/10.2214/AJR.17.18020.

8. Frenkel WJ, Jongerius EJ, Mandjes-van UM, et al. Validation of the Charlson Comorbidity Index in acutely hospitalized elderly adults: a prospective cohort study. J Am Geriatr Soc. 2014;62(2):342-6. https://doi.org/10.1111/ jgs.12635.

9. Amin MB, Greene FL, Edge SB, et al. The eighth edition AJCC cancer staging manual: continuing to build a bridge from a population-based to a more personalized approach to cancer staging. CA Cancer J Clin. 2017;67(2):93-9. https://doi.org/10.3322/caac.21388.

10. Mitropoulos D, Artibani W, Biyani CS, et al. Validation of the Clavien-Dindo grading system in urology by the European association of urology guidelines ad hoc panel. Eur Urol Focus. 2018;4(4):608-13. https://doi.org/10. 1016/j.euf.2017.02.014

11. Zhuang C, Huang D, Pang W, et al. Sarcopenia is an independent predictor of severe postoperative complications and long-term survival after radical gastrectomy for gastric cancer: analysis from a large-scale cohort. Medicine. 2016;95(13):e3164. https://doi.org/10.1097/MD.0000000000003164.

12. Cruz-Jentoft AJ, Sayer AA. Sarcopenia. Lancet. 2019;393(10191):2636-46. https://doi.org/10.1016/S0140-6736(19)31138-9.

13. Prado CM, Lieffers JR, McCargar $L$, et al. Prevalence and clinical implications of sarcopenic obesity in patients with solid tumours of the respiratory and gastrointestinal tracts: a population-based study. Lancet Oncol. 2008;9(7):629-35. https://doi.org/10.1016/S1470-2045(08)70153-0.

14. Nishigori T, Obama K, Sakai Y. Assessment of body composition and impact of sarcopenia and sarcopenic obesity in patients with gastric cancer. Transl Gastroenterol Hepatol. 2020:5:22. https://doi.org/10.21037/tgh.2019.10.13.

15. Schizas D, Frountzas M, Lidoriki I, et al. Sarcopenia does not affect postoperative complication rates in oesophageal cancer surgery: a systematic review and meta-analysis. Ann R Coll Surg Engl. 2020;102(2):120-32. https:// doi.org/10.1308/rcsann.2019.0113.

16. Nishimura JM, Ansari AZ, Souza DM, et al. Computed tomography-assessed skeletal muscle mass as a predictor of outcomes in lung cancer surgery. Ann Thorac Surg. 2019;108(5):1555-64. https://doi.org/10.1016/j.athoracsur. 2019.04.090.

17. Guo Z, Gu C, Gan S, et al. Sarcopenia as a predictor of postoperative outcomes after urologic oncology surgery: a systematic review and metaanalysis. Urol Oncol. 2020;38(6):560-73. https://doi.org/10.1016/j.urolonc. 2020.02.014.

18. Ouellette JR, Small DG, Termuhlen PM. Evaluation of Charlson-age comorbidity index as predictor of morbidity and mortality in patients with colorectal carcinoma. J Gastrointest Surg. 2004;8(8):1061-7. https://doi.org/ 10.1016/j.gassur.2004.09.045.

19. Miller SL, Wolfe RR. The danger of weight loss in the elderly. J Nutr Health Aging. 2008;12(7):487-91. https://doi.org/10.1007/BF02982710.

20. Morgan PT, Smeuninx B, Breen L. Exploring the impact of obesity on skeletal muscle function in older age. Front Nutr. 2020;7:569904. https://doi.org/10. 3389/fnut.2020.569904.

21. Fujiwara N, Nakagawa H, Kudo Y, et al. Sarcopenia, intramuscular fat deposition, and visceral adiposity independently predict the outcomes of hepatocellular carcinoma. J Hepatol. 2015;63(1):131-40. https://doi.org/10. 1016/j.jhep.2015.02.031.

22. Yoon SL, Kim JA, Kelly DL, et al. Predicting unintentional weight loss in patients with gastrointestinal cancer. J Cachexia Sarcopenia Muscle. 2019;10(3):526-35. https://doi.org/10.1002/jcsm.12398.

23. $\mathrm{Ni} \mathrm{J}$, Zhang L. Cancer Cachexia: definition, staging, and emerging treatments. Cancer Manag Res. 2020;12:5597-605. https://doi.org/10.2147/ CMAR.S261585.

\section{Publisher's Note}

Springer Nature remains neutral with regard to jurisdictional claims in published maps and institutional affiliations. 